\title{
¿QuiÉn CUIDA en la CIUDAD? Aportes Para políticas
}

\section{URBANAS DE IGUALDAD}

\author{
Who assumes care in the city? Contributions for urban policies of \\ equality
}

\section{Olga Elena Segovia Marín ${ }^{1}$}

Fecha de recepción: 06-04-2018 - Fecha de aceptación: 16-05-2018

Hábitat y Sociedad (ISSN 2173-125X), n. ${ }^{\circ}$ 11, noviembre de 2018, pp. 257-263.

http://dx.doi.org/10.12795/HabitatySociedad.2018.i11.16

\begin{abstract}
The book ¿Quién cuida en la ciudad? Aportes para políticas urbanas de igualdad (Who assumes care in the city? Contributions for urban policies of equality) participates in the debate on the construction of inclusive cities with gender equality taking stand from the perspective of care. It postulates that such a goal implies overcoming the dichotomous visions that separate the public and the private, to conceive the urban as a space that articulates production and consumption, and reproduction of everyday life.

The book presents the results of recent studies carried out by the Economic Commission for Latin America and the Caribbean (ECLAC) in Latin American cities. They are united by a common proposal: care is a political issue, as is the autonomy of women, an aspect closely linked to care. It is necessary, therefore, to address them with public policies that generate changes in a social structure where domestic and care work is assigned primarily to women. Being not remunerated, it is closely associated to the poverty of time and space that affects them.

The proposal of the book is summarized in indicating the road towards a "caretaker city", where, in a context of co-responsibility and solidarity, each and every one cares: the State, the market, families, the community, men and women
\end{abstract}

Key words

Equality; Gender; Cities; Care

\section{Resumen}

El libro ¿Quién cuida en la ciudad? Aportes para políticas urbanas de igualdad se inserta, desde la perspectiva del cuidado, en el debate sobre la construcción de ciudades inclusivas con igualdad de género. Postula que tal meta implica superar las visiones dicotómicas que separan lo público y lo privado, para concebir lo urbano como un espacio que articula producción y consumo y reproducción de la vida cotidiana.

El libro recoge los resultados de estudios recientemente realizados por la Comisión Económica para América Latina y el Caribe (CEPAL) en ciudades latinoamericanas. Los une una propuesta común: el cuidado es asunto político, como también lo es la autonomía de las mujeres, aspecto estrechamente ligado a los cuidados. Se requiere, entonces, abordarlos con políticas públicas que generen cambios en una estructura social donde el trabajo doméstico y de cuidados se asigna prioritariamente a las mujeres. No es remunerado, lo que incide centralmente en la pobreza de tiempo y espacio que las afecta.

La propuesta del libro se resume en indicar el camino hacia una "ciudad cuidadora", donde, en un contexto de corresponsabilidad y solidaridad, todos cuidan: el Estado, el mercado, las familias, la comunidad, los hombres y las mujeres.

\section{Pallabras clave}

Igualdad; Género; Ciudades; Cuidado

1 Arquitecta e Investigadora de la División Asuntos de Género de la CEPAL. E-mail: olga.segovia@un.org. 
Nieves Rico, M. y Segovia, O. (eds.) (2017). ¿Quién cuida en la ciudad? Aportes para políticas urbanas de igualdad. Santiago (Chile): Naciones Unidas, Comisión Económica para América Latina y el Caribe (CEPAL). ISBN: 978-92-1-121970-8

\section{Seminario Internacional: Quién cuida en la ciudad? Políticas} urbanas y autonomía económica de las mujeres. Santiago, Chile, CEPAL, 21-22 de noviembre de 2017

El pasado mes de noviembre de 2017, CEPAL organizó en Santiago de Chile el Seminario Internacional ¿Quién cuida en la ciudad? Políticas urbanas y autonomía económica de las mujeres, evento desarrollado en el marco del proyecto Desarrollo urbano, autonomía económica de las mujeres y cuidado, implementado por la División de Asuntos de Género de la CEPAL, con el apoyo de la Cuenta para el Desarrollo de las Naciones Unidas, en diversas ciudades de América Latina. A él asistieron autoridades de gobierno, expertas y representantes de la sociedad civil de ciudades de América Latina.

Un aspecto central de este Seminario fue la presentación del libro ¿Quién cuida en la ciudad? Aportes para políticas urbanas de igualdad, editado por María Nieves Rico y Olga Segovia y publicado por CEPAL en 2017.

El propósito de este Seminario, y del libro, fue contribuir al debate y a la formulación de políticas urbanas con igualdad de género, en el contexto de los principios y lineamientos de la Agenda Regional de Género (CEPAL, 2017a) y la Estrategia de Montevideo (CEPAL, $2017 b)$. La propuesta discutida -tanto en el libro como en el seminario- fue la de una ciudad cuidadora, en la cual mujeres y hombres puedan usar y disfrutar del tiempo y del espacio de la ciudad en condiciones de igualdad y en ejercicio de sus derechos, avanzando hacia la superación de la situación actual, en que la mujer asume la mayor parte de las tareas de cuidado al interior de la familia en las ciudades. Concretamente, se indaga en quiénes cuidan y quiénes son cuidados en las ciudades latinoamericanas. Sabiendo que es una tarea asignada culturalmente a las mujeres, el libro aporta en materia de propuestas que les faciliten el ejercicio de sus derechos y la ampliación de su autonomía.

Para las autoras, el tema del cuidado debe ser abordado desde la pobreza de tiempo y de espacio que afecta a las mujeres y que se vincula al actual modelo de desarrollo de la ciudad y de los países. El punto de partida para ello es el reconocimiento de la función social del cuidado, ignorada y poco valorada en nuestras sociedades. Esta situación se hace eco de una sociedad que solo valora el trabajo productivo, remunerado, pasando por alto la necesidad del trabajo reproductivo como sostén mismo de la producción.

$\mathrm{Al}$ respecto, una inquietud inevitable es el futuro del cuidado en sociedades como las latinoamericanas, con una urbanización estable y consolidada y una población urbana que envejece, y en donde la escasez de servicios de cuidado institucionalizados hace que las tareas de cuidado — de la niñez, ancianidad y personas con discapacidades- recaiga principalmente en las familias, y por tanto en las mujeres. Como se destaca en el libro, este es asunto político. Su magnitud exigirá un cambio cultural, relativo al compartir los trabajos de cuidados no solo entre hombres y mujeres al interior del hogar, sino en la sociedad en- 
tera; y un cambio político, materializado en una forma distinta de organización de la sociedad.

El libro destaca un punto no habitual en los estudios urbanos, como es el hecho de que mujeres y hombres habitan la ciudad de manera distinta. Indagar en quienes cuidan y son cuidados y en cómo la ciudad puede ser pensada, construida y gestionada para acoger las necesidades de aquellas personas a quienes se les ha asignado culturalmente las tareas de cuidado, es el camino recorrido en este libro. Sin embargo, se señala el horizonte simbólico de las ciudades como lugares de igualdad de oportunidades, de resultados y de derechos, que enfrenta límites y desafíos en los que a menudo se cruzan las brechas socioeconómicas y de género.

Los contenidos del presente libro, aquí sintetizados, están organizados temáticamente en tres partes.

En la primera parte, "Habitar la ciudad con igualdad de género", se propone un marco conceptual sobre aspectos del desarrollo urbano que son clave para un paradigma que integre la igualdad de género. En particular, se considera el desafío que enfrentan las ciudades en América Latina y el Caribe respecto a la urbanización, planificación y gestión de las ciudades y el uso del tiempo y del cuidado.

En el primer capítulo, Olga Segovia y María Nieves Rico resaltan el hecho de que el espacio de las ciudades no es neutro. Se encuentra cruzado por brechas económicas y sociales y su ocupación y uso son marcadamente diferentes según se trate de mujeres o de hombres. La planificación urbana debe expresar y responder a tales diferencias. Esto es, se requiere una forma distinta de planificar y gestionar la ciudad que se haga cargo de las necesidades de cuidado; que reconozca el aporte que realizan las mujeres con el trabajo no remunerado al interior de los hogares y que contribuya a su autonomía económica, otorgando los servicios e infraestructuras necesarias para ello y promoviendo una cultura de corresponsabilidad urbana de la vida cotidiana.

Antonio Prado y Vera Kiss, en el segundo capítulo, sitúan la discusión de las desigualdades distinguiendo la primacía urbana de América Latina y el Caribe y destacando que es una región con una alta tasa de urbanización consolidada. En esa perspectiva, señalan la importancia de las ciudades como territorios privilegiados para la aplicación de políticas y programas públicos destinados a superar las desigualdades estructurales que caracterizan a la región, a la vez que permitan un desarrollo sostenible.

En el capítulo III, María-Ángeles Durán explora un nuevo paradigma: ciudades que cuidan. Para ello, revisa diferentes formas de cuidado y, aplicándolo al caso de Argentina, estima el valor de la producción de cuidado doméstico no remunerado y sus costos de sustitución. De esta manera hace patente la enorme magnitud del trabajo de cuidado no remunerado efectuado principalmente por mujeres. Y advierte que, en el futuro próximo, es improbable que las familias puedan hacerse cargo de los nuevos requerimientos de cuidado de la población mayor y serán necesarias grandes reformas organizativas para dar solución a la demanda insatisfecha. Puesto así, las tareas de cuidado salen de la invisibilidad del espacio privado, situándose como un asunto político.

En el cuarto capítulo, Lucía Scuro e Iliana Vaca-Trigo destacan la importancia de las mediciones del uso, distribución y asignación del tiempo desde una perspectiva de género, y analizan los resultados de encuestas del uso del tiempo relativas al trabajo remunerado y no remunerado — doméstico y de cuidados- en nueve ciudades capitales 
latinoamericanas. Sus conclusiones muestran que la creciente incorporación de las mujeres al mercado laboral no ha ido de la mano con una mayor participación de los hombres en las tareas domésticas y de cuidado de los hogares, lo que presenta nuevos retos a las políticas urbanas sociales y económicas.

En la segunda parte del libro, "Autonomía económica de las mujeres y cuidados en ciudades latinoamericanas", se presentan los principales hallazgos y resultados de los estudios realizados en siete ciudades de la región que forman parte del Proyecto de la Cuenta de Naciones Unidas para el "Desarrollo urbano, autonomía económica de las mujeres y cuidado", implementado por la División Asuntos de Género de CEPAL. En ellos se aborda la importancia de la integración de la perspectiva de género en el desarrollo urbano, con un enfoque específico en la autonomía económica y la redistribución del trabajo de cuidado. Se considera desde la gran área metropolitana que es Ciudad de México (México), con más de veinte millones de habitantes, a áreas metropolitanas como Bogotá (Colombia), ciudades capitales como Montevideo (Uruguay), ciudades de nivel intermedio como Cuenca (Ecuador) y Rosario (Argentina), y la comuna de Santiago de Chile, un caso de gestión local en el centro político de la ciudad y del país.

A la pregunta central, ¿quién cuida en la ciudad?, la respuesta en todos los casos examinados es unánime: las mujeres, y ello mayoritariamente al interior de sus hogares e independientemente de su situación en el mercado laboral. Ello hace referencia a la ausencia de políticas públicas de cuidado, a la segmentación de la oferta de mercado y a la resistencia de los hombres a tener un rol más activo en la reproducción social. Asimismo, los resultados respecto a la autonomía económica de las mujeres son similares. La constatación es unánime: la división sexual del trabajo se mantiene, la producción social es masculina y la reproducción social, femenina.

En el capítulo V, desde la economía feminista, Lucía Pérez Fragoso resalta la relevancia que presentan para los análisis y propuestas los conceptos de autonomía económica y políticas de cuidados. En esta línea, la autonomía económica de las mujeres se refiere a algo más amplio que el acceso a un empleo remunerado o a ingresos propios e implica la oportunidad de negociar las cargas de trabajo doméstico no remunerado. Al respecto, la autora revisa los programas vigentes en la Ciudad de México y las competencias del gobierno local en distintos ámbitos vinculados al cuidado y realiza un análisis prospectivo del aumento de la demanda de cuidados, para proponer programas y proyectos en la materia a corto, mediano y largo plazo.

En el sexto capítulo, y en la misma dirección, el texto de Amalia García Medina expone la importancia de la propuesta del movimiento feminista de considerar al cuidado como un bien público y como un derecho, que se incorporó en la Constitución de la Ciudad de México, aprobada en 2017. En ella se reconoce el derecho al cuidado al señalar expresamente: "Toda persona tiene derecho al cuidado que sustente su vida y le otorgue los elementos materiales y simbólicos para vivir en sociedad a lo largo de toda su vida" (Constitución Política de la Ciudad de México, 2017, art. 9. B). Presenta, además, los principales avances de la Nueva Cultura Laboral de la Ciudad de México vinculados al cuidado, que incluyen la reducción de la jornada de trabajo, la ampliación de licencias maternales y paternales y la asignación de tiempo en el lugar de trabajo para estudiar. 
Karina Batthyány, en el capítulo VII dedicado a la ciudad de Montevideo, destaca la importancia de que las políticas públicas contemplen la carga de trabajo doméstico y de cuidado no remunerado de las mujeres, puesto que solo contemplan la realidad de aquellas que están incorporadas a la fuerza de trabajo. En el texto se hace un llamado a promover y facilitar la descentralización y territorialización de la política nacional de cuidados.

Olga Segovia, en el capítulo VIII acerca de la comuna de Santiago, propone que el desarrollo urbano debe ser pensado según dos conceptos básicos: el enfoque territorial y la perspectiva de género. Sostiene que no se puede hablar de autonomía económica de las mujeres en abstracto sin considerar el contexto físico y social en que están situadas: la vivienda, el barrio y la ciudad. Y señala que abordar la planificación de los servicios de cuidado -la cual incluye identificar prioridades de localización y de organización del tiempo de atención de estos servicios- desde un enfoque de género permite profundizar en una visión territorial.

La movilidad y la inseguridad en la ciudad son dos temas relevantes para la autonomía de las mujeres en la ciudad, según señala Marisol Dalmazzo en el capítulo IX sobre Bogotá. La separación entre los lugares de vivienda y los de trabajo, los sistemas de transporte organizados desde una perspectiva del trabajo de los hombres o los equipamientos distantes limitan las posibilidades laborales de las mujeres. Y a ellos se suma la violencia contra ellas en el espacio público, que limita su autonomía por la percepción de inseguridad. En el texto se expone un conjunto de propuestas que comprenden la estructuración del Sistema Distrital de Cuidado en Bogotá y el desarrollo de políticas transversales que incorporan la dimensión de género.

En el capítulo X sobre la ciudad de Rosario, Ana Falú señala que las mujeres relegadas al mundo privado permanecen invisibles para quienes piensan las ciudades. Al respecto indica que la incorporación de la perspectiva de género y las reivindicaciones del movimiento feminista -identidad, autonomía económica y participación en las políticas de las ciudades- son centrales. Sostiene que, en el tema de las políticas de cuidado y de la autonomía de las mujeres en las ciudades, coinciden dos perspectivas: la de los derechos de las mujeres que cuestionan la división sexual del trabajo y la del derecho a la ciudad en cuanto a la omisión que ha existido de las mujeres y de la perspectiva de género en el urbanismo. El texto reconoce avances en la ciudad de Rosario en torno a políticas públicas y derechos de las mujeres.

Morena Herrera, en el capítulo XI dedicado a la ciudad de San Salvador, destaca que, para pensar las ciudades desde la perspectiva de quienes cuidan, el punto de partida es el reconocimiento de la función social del cuidado como labor esencial para la vida, y cuya satisfacción se vincula al avance en la autonomía económica de las mujeres. El texto plantea la necesidad de la generación de información sobre la demanda de cuidado en la ciudad de San Salvador, que permita establecer lineamientos y medidas de política pública que contribuyan a la creación de un sistema nacional y municipal de cuidados.

En el último capítulo de la segunda parte del libro (Capítulo XII), sobre la ciudad de Cuenca, Ecuador, se propone la inclusión de una perspectiva de género en las iniciativas locales que impulsan emprendimientos económicos, considerando entregar servicios de cuidados, favorecer la autonomía económica de las mujeres y, junto con ello, for- 
talecer la gestión e institucionalización del enfoque de género en la Municipalidad de Cuenca.

En la tercera parte, "Desafíos para la vida cotidiana: Movilidad urbana y uso del tiempo", se exploran las tensiones que se presentan entre la búsqueda de la autonomía económica de las mujeres y las tareas de cuidado; tensiones que se expresan en las diferentes estrategias de movilidad que las mujeres que buscan una autonomía económica despliegan en la ciudad y en el uso del tiempo.

Paola Jirón, en el capítulo XIII, indaga en las estrategias que despliegan las mujeres para organizar su propia movilidad y la de los integrantes de sus hogares en la ciudad. Ello le permite concluir que las políticas públicas no han considerado la diversidad de experiencias de movilidad que conviven en la ciudad y que éstas no se acaban en el transporte público. Al respecto menciona temas de movilidad que se mantienen pendientes, relacionados con la coordinación territorial de los sistemas educacional, de salud, laboral y comercial, los cuales se deben ajustar a las necesidades de la vida cotidiana.

Laura Pautassi, en el capítulo XIV, aborda la movilidad de los niños, niñas y adolescentes y las estrategias de cuidado correspondientes. A partir de los resultados de una encuesta sobre movilidad escolar en la Región Metropolitana de Buenos Aires, destaca que los desplazamientos estudiados, y en general en las situaciones de cuidado, se requiere de un(a) "acompañante" que asume directamente el costo de tiempo de la movilidad. Esto implica una modificación de los patrones de viaje habituales del acompañante para "acomodarse" a las necesidades de la persona acompañada.

En el capítulo XV de conclusiones, "Hacia ciudades cuidadoras", María Nieves Rico y Olga Segovia plantean que la ciudad cuidadora es un escenario y un actor donde todos cuidan; donde el Estado, el mercado, las familias y la comunidad, así como los hombres y las mujeres, comparten la responsabilidad del cuidado en una nueva organización social. Una ciudad cuidadora e inclusiva incorpora un urbanismo cuyas políticas y modelos de gestión respondan a los desafíos de sociedades en creciente transformación demográfica, socioeconómica, social y tecnológica. Se trata, por tanto, de reconocer y promover la centralidad del cuidado en la vida colectiva y concebir ciudades cuya planificación y gestión expresen una sociedad que incluye la diversidad de sus habitantes.

Este capítulo, y el libro, se cierran con la convicción de que una ciudad inclusiva y cuidadora "es una ciudad no neutral y profundamente política, que basa su devenir en la convicción de que transformar el espacio y el tiempo es transformar la realidad" (p. 469). 


\section{Referencias}

CEPAL (2017a). 40 años de Agenda Regional de Género. Santiago de Chile: Naciones Unidas. Recuperado el 04 de abril de 2018, de: https:/ / www.cepal.org/ es/publicaciones/40333-40-anos-agenda-regionalgenero.

CEPAL (2017b). Estrategia de Montevideo para la implementación de la Agenda Regional de Género en el Marco del Desarrollo Sostenible. Santiago de Chile: Naciones Unidas. Recuperado el 04 de abril de 2018, de: https://www.cepal.org/es/publicaciones/41011-estrategia-montevideo-la-implementacion-la-agendaregional-genero-marco.

Asamblea Constituyente de la Ciudad de México
(2017, 30 de enero). Constitución Política de Ciudad de México. Ciudad de México: Asamblea Constituyente de la Ciudad de México. Recuperado el 18 de abril de 2018, de: http:/ /infodf.org.mx/documentospdf/constitucion_cdmx/Constitucion_\%20Politica_CDMX.pdf.

Rico, M. N. y Segovia, O. (eds.) (2017). ¿Quién cuida en la ciudad? Aportes para políticas urbanas de igualdad. Santiago de Chile: Naciones Unidas / CEPAL. Recuperado el 17 de julio de 2018, de: https:/ / www.cepal.org/sites/default/files/events/files/ quien_cuida_en_la_ciudad._aportes_para_politicas_urbanas_de_igualdad.pdf.

Segovia Marin, O.E. (2018). ¿Quién cuida en la ciudad? Aportes para políticas urbanas de igualdad. Hábitat y Sociedad, 11, 257263.

<http://dx.doi.org/10.12795/HabitatySociedad.2018.i11.16> 


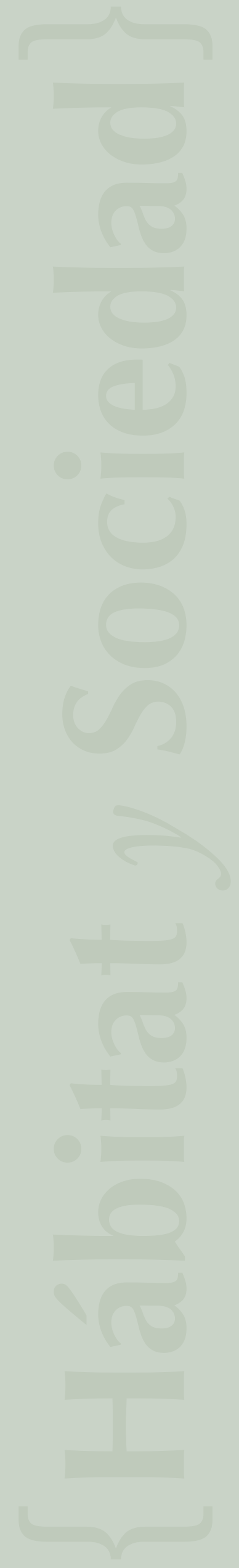

